\title{
Guest-editorial
}

\section{A journey in Computational Logic in Italy}

\author{
Matteo Baldoni* and Cristina Baroglio \\ Dipartimento di Informatica, Università degli Studi di Torino, Torino, Italy
}

This issue reviews the recent work of the Italian research groups, whose activity focuses on the use of methodologies and of techniques that are based on computational logic, a fundamental research area of Artificial Intelligence.

The study of computational logic has a long tradition in Italy. GULP (the name of the Italian Association for Logic Programming), with its 25 years of existence, is the oldest national association ever founded on this topic, even older than the international Association for Logic Programming. It was, in fact, founded in 1985 , soon raising to the highest international levels and becoming the promoter of initiatives that provided both educational and scientific opportunities to many young researchers.

After 2004, researchers realized that the time was mature for opening to neighboring and applicative fields and to start proposing logic programming also as a tool for solving problems of interest to other disciplines. The aim of this special issue is to witness this effort. As such, it complements the survey of the first twenty-five years of existence of GULP, edited by Agostino Dovier and Enrico Pontelli in 2010. The fifteen selected articles, included in the special issue, describe some of the most relevant recent experiences and some of the most promising investigations that were (and are being) carried on in such diverse fields as the (Semantic) Web, the specification and verification of interaction proto-

\footnotetext{
*Corresponding author. Dipartimento di Informatica, Università degli Studi di Torino, c.so Svizzera 185, I-10149 Torino, Italy. E-mails: baldoni@di.unito.it (M. Baldoni); baroglio@di.unito.it (C. Baroglio).
}

cols, bioinformatics, intelligent agents, non-monotonic reasoning, business processes, program transformation, non-classical logics and knowledge representation, and constraint programming. On the whole, forty-eight researchers contributed to the realization of this special issue of the journal.

The Special Issue is dedicated to Prof. Alberto Martelli, who is well-known in the Artificial Intelligence community, and in particular in the field of computational logic. The career of prof. Martelli is strictly tied to the development of Computer Science in Italy. After getting his degree from the Politecnico of Milan, he moved to Pisa, where the first curriculum in Computer Science was being organized, and a new research group on Computer Science was being formed at the local offices of the National Research Council (Istituto di Elaborazione dell'Informazione). The early years were devoted to the study of image processing but soon the interest of the group moved towards the emerging field of Artificial Intelligence. Long research visits in the USA gave the members of the group an up-to-date view of the cutting-edge research topics. In particular, Alberto Martelli spent one year at the Electrical Engineering and Computer Science Department of the New York University, in New York. In those years his studies concerned the foundations of dynamic programming and, in particular, the optimal smoothing in image processing by means of dynamic programming [15, 20]. Later, he faced optimization problems by means of Artificial Intelligence methods, like finding minimal cost solutions in AND-OR trees. The results deserved presentation at the best conferences, like IJCAI [21, 22], and publication in the best journals, like Artificial Intel- 
ligence [18], Communications of ACM [17,23], and the Journal of ACM [14, 16]. From 1976, his researches moved to the emerging area of logic programming, with a particular interest in unification as the central step of resolution, which was (and is) used by Prolog interpreters. The original formulation was quite inefficient. Alberto Martelli, together with Ugo Montanari, developed a linear algorithm presented as a Technical Report in the same year, later reformulated as a nondeterministic algorithm, from which the efficient one is derived [24].

Since November 1981 he is full professor of Computer Science at the University of Torino, where he was director of the Dipartimento di Informatica from 1984 to 1989 , and chairman of the PhD program in Computer Science from 1988 to 1995 . Here he founded the Logic Programming and Automated Reasoning research group. In those years, his research activity was focused on pattern-matching techniques for logic languages, that were based on the unification algorithm, and proposed variants of the latter that could tackle infinite terms $[19,25,26]$. Later on, the group became interested also in non-monotonic reasoning, in particular in the characterization of Truth Maintenance Systems, in the properties of default logics, and in negation by failure in logic programming $[5,8,11]$.

In the late ' 80 s the group began working on non-classical logics, in particular modal logic, studying extensions of logic programming languages with structuring constructs, that are typical of traditional programming languages - such as blocks and modules $[6,7,10]$, and with belief revision capabilities, which allow dealing with hypothetical and non-monotonic reasoning $[3,4]$. Later on the activity focused on the study of techniques for reasoning about actions and change, mainly by making use of modal and temporal logic: in particular, a theory of actions [12], based on a combination of temporal and of dynamic logic, was developed together with a modal logic programming language to reason about actions [2]. These were, then, applied to the area of specification and verification of properties of multiagent systems and web services, with the goal of using the above mentioned logic-based formalisms and tools to specify agent interactions and to prove their properties $[1,9,13]$.

He was head of a group in the ESPRIT BRA Project MEDLAR I and II "Mechanizing Deduction in the Logics of Practical Reasoning" (1989-95), coordinator of some national projects and representative of the Turin group in the VI framework Network of Excellence REWERSE (Reasoning on the web with rules and semantics). He is a fellow of ECCAI, the European Artificial Intelligence Association, since 2005.

Besides his scientific merits, the guest editors would like to thank Alberto for his kindness and for the benevolence he always showed to his collaborators and to his students.

\section{Acknowledgements}

The guest editors would like to thank all the people who contributed to this special issue: the authors and the reviewers and, especially, Laura Giordano, Evelina Lamma, Paola Mello, Nicola Olivetti, Viviana Patti, Maria Luisa Sapino, and Piero Torasso for their encouragement and support, Oliviero Stock and the editorial board the Intelligenza Artificiale journal for the opportunity of realizing this special issue.

\section{References}

[1] M. Baldoni, C. Baroglio, A. Martelli and V. Patti, Reasoning about interaction protocols for customizing web service selection and composition, Journal of Logic and Algebraic Programming 70(1) (2007), 53-73.

[2] M. Baldoni, L. Giordano, A. Martelli and Patti, Programming rational agents in a modal action logic, Annals of Mathematics and Artificial Intelligence 41(2-4) (2004), 207-257.

[3] D.M. Gabbay, L. Giordano, A. Martelli and N. Olivetti, A language for handling hypothetical updates and inconsistency, Logic Journal of the IGPL 4(3) (1996), 385-416.

[4] D.M. Gabbay, L. Giordano, A. Martelli, N. Olivetti and M.L. Sapino, Conditional reasoning in logic programming, Journal of Logic Programming 44(1-3) (2000), 37-74.

[5] L. Giordano and A. Martelli, On cumulative default logics, Artificial Intelligence 66(1) (1994), 161-179.

[6] L. Giordano and A. Martelli, Structured Prolog: A language for structured logic programming, Software-Concepts and Tools 15(3) (1994), 125-145.

[7] L. Giordano and A. Martelli, Structuring logic programs: a modal approach, Journal of Logic Programming 21(2) (1994), 59-94.

[8] L. Giordano and A. Martelli, A logical characterization for truth maintenance systems with dependency-directed backtracking, Computational Intelligence 11 (1995), 11-46.

[9] L. Giordano and A. Martelli, Tableau-based automata construction for dynamic linear time temporal logic*, Annals of Mathematics and Artificial Intelligence 46(3) (2006), 289315.

[10] L. Giordano, A. Martelli and G. Rossi, Extending horn clause logic with implication goals, Theoretical Computer Science 95(1) (1992), 43-74.

[11] L. Giordano, A. Martelli and M.L. Sapino, Extending negation as failure by abduction: a three-valued stable model semantics, Journal of Logic Programming 26(1) (1996), 31-67.

[12] L. Giordano, A. Martelli and C. Schwind, Reasoning about actions in dynamic linear time temporal logic, Logic Journal of the IGPL 9(2) (2001), 273-288. 
[13] L. Giordano, A. Martelli and C. Schwind, Specifying and verifying interaction protocols in a temporal action logic, Journal of Applied Logic 5(2) (2007), 214-234.

[14] S. Gnesi, A. Martelli and U. Montanari, Dynamic programming as graph searching: an algebraic approac, Journal of the ACM 28(4) (1981), 737-751.

[15] A. Martelli, Edge detection using heuristic search methods, Computer Graphics and Image Processing 1(2) (August 1972), 169-182.

[16] A. Martelli, A gaussian elimination algorithm for the enumeration of cut sets in a graph, Journal of the ACM 23(1) (1976), $58-73$.

[17] A. Martelli, An application of heuristic search methods to edge and contour detection, Communications of the ACM 19(2) (February 1976), 73-83.

[18] A. Martelli, On the complexity of admissible search algorithms, Artificial Intelligence 8(1) (February 1977), 1-13.

[19] A. Martelli, C. Moiso and G. Rossi, Al algorithm for unification in equational theories, in: Proc of Symposium on Logic Programming, Salt Lake City, UT, 1986, pp. 180-186.

[20] A. Martelli and U. Montanari, Nonserial dynamic programming: on the optimal strategy of variable elimination for the rectangular lattice, Journal of Mathematical Analysis and Applications 40(1) (October 1972), 226-242.

[21] A. Martelli and U. Montanari, Additive AND-OR graphs, in: Proc. of 3rd International Joint Conference on Artificial Intelligence, Stanford, CA, August 20-23, 1973, pp. 1-11.

[22] A. Martelli and U. Montanari, From dynamic programming to search algorithms with functional costs, in: Proc. of $3 \mathrm{rd}$ International Joint Conference on Artificial Intelligence, Tbilisi, September 3-8, 1975, pp. 345-350.

[23] A. Martelli and U. Montanari, Optimizing decision trees through heuristically guided search, Communications of the ACM 21(12) (December 1978), 1025-1039.

[24] A. Martelli and U. Montanari, An efficient unification algorithm. ACM Transactions on Programming Languages and Systems 4(2) (April 1982), 258-282.

[25] A. Martelli and G. Rossi, Efficient unification with infinite terms in logic programming, in: Proc of Int Conference on Fifth Generation Computer Systems, Tokyo, Japan, November 1984, pp. 202-209.

[26] A. Martelli and G. Rossi, Stepwise development of an algorithm for unification over infinite terms, Computers and Artificial Intelligence 9(3) (1990), 209-239. 